\title{
Evaluation of the prognostic ability of serum uric acid for elderly acute coronary syndrome patients with diabetes mellitus: a prospective cohort study
}

\author{
Yang JIAO ${ }^{1 *}$, Jihang $\mathrm{WANG}^{1,2^{*}}$, Xia YANG ${ }^{1}$, Mingzhi SHEN ${ }^{2}$, Hao XUE ${ }^{1}$, Jun GUO ${ }^{1}$, Wei DONG ${ }^{1}$, \\ Yundai CHEN ${ }^{1}$, Qing XI ${ }^{3 凶}$, Zhenhong $\mathrm{FU}^{1 凶}$ \\ ${ }^{1}$ Senior Department of Cardiology, the Sixth Medical Center of PLA General Hospital \& Chinese PLA Medical School, Beijing 100853, China \\ ${ }^{2}$ Department of Cardiology, Hainan Hospital, Chinese PLA General Hospital, Sanya 572000, China \\ ${ }^{3}$ The First Medical Center, Chinese PLA General Hospital, Beijing 100853, China
}

\begin{abstract}
Objective: This study evaluated the prognostic power of serum uric acid (UA) in predicting adverse events in elderly acute coronary syndrome (ACS) patients with diabetes mellitus (DM). Methods: The analysis involved 718 ACS patients $>80$ years old whose general clinical data and baseline blood biochemical indicators were collected prospectively from January 2006 to December 2012. These patients were classified into two groups based on DM status, and then followed up after discharge. The Kaplan-Meier method was used for major adverse cardiac event (MACE) rates and all-cause mortality. Multivariate Cox regression was performed to analyze the relationship between UA level and long-term clinical prognosis. Receiver operating characteristic (ROC) curves were analyzed to predict the cutoff value of UA in elderly ACS patients with DM. There were 242 and 476 patients in the DM and non-DM (NDM) groups, respectively, and the follow-up time after discharge was 40-120 months (median, 63 months; interquartile range, 51-74 months). Results: The all-cause mortality, cardiac mortality, and MACE rates in both DM and NDM patients were higher than those in the control group $(P=0.001)$. All-cause mortalities, cardiac mortalities, and MACE rates in DM patients with moderate and high UA levels were significantly higher than those in the NDM group $(P=0.001)$. Long-term survival rates decreased significantly with increased UA levels in the ACS groups $(P=0.001)$. UA (odds ratio $(\mathrm{OR})=2.106,95 \%$ confidence interval $(\mathrm{CI})=1.244-3.568, P=0.006$ ) was found to be an independent risk factor for all-cause mortality and MACE in elderly ACS patients with DM. The cutoff value of UA was $353.6 \mu \mathrm{mol} / \mathrm{L}$ (sensitivity, $67.4 \%$; specificity, 65.7\%). Conclusions: Serum UA level is a strong independent predictor of long-term all-cause death and MACE in elderly ACS patients with DM.
\end{abstract}

Key words: Uric acid; Elderly patient; Acute coronary syndrome; Diabetes mellitus; Prognosis

\section{Introduction}

According to the 2020 China cardiovascular disease report, the population with cardiovascular disease in China has reached 330 million, with cardiovascular diseases as the leading causes of death in China, including 11.39 million patients with coronary heart disease (CHD) (National Center for Cardiovascular Diseases, 2021).

\footnotetext{
$\triangle$ Zhenhong FU, fuzhenh@126.com Qing XI, xiqing301@163.com

* The two authors contributed equally to this work

(iD) Zhenhong FU, https://orcid.org/0000-0002-2784-7629

Qing XI, https://orcid.org/0000-0002-3422-7356
}

Received Oct. 13, 2020; Revision accepted May 18, 2021; Crosschecked Sept. 2, 2021

(C) Zhejiang University Press 2021
Acute coronary syndrome (ACS) is a clinical subtype of $\mathrm{CHD}$, which is a group of clinical syndromes caused by acute myocardial ischemia. The clinical classification includes unstable angina pectoris, acute ST-segment elevation myocardial infarction (STEMI), and acute nonST-segment elevation myocardial infarction (NSTEMI) (Komaru et al., 2019). Important mechanisms for the occurrence of ACS include microcirculation disorder, type of atherosclerotic plaque, function of vascular endothelium, decrease of coronary blood flow, and inflammatory response (Jin et al., 2017). Old age and diabetes mellitus (DM) are both independent risk factors for ACS (Guo et al., 2018). It is reported that about $75 \%$ of patients hospitalized for CHD have abnormal glucose metabolism, and the rate of coronary artery 
lesions is relatively high (Fu et al., 2013; Bonaca et al., 2018). Persistent hyperglycemia in patients results in disorders of platelet structure and function, enhances aggregation and adhesion of platelets, and increases the risk of thrombosis (Xu et al., 2020). Uric acid (UA) is the final product of purine oxidation metabolism in the human body and is excreted with urine. Clinical studies illustrate that the patients with conditions such as CHD, hypertension, heart failure, DM, metabolic syndrome, or chronic renal insufficiency have high serum UA levels, and high UA in the body not only increases the risk of cardiovascular disease, but also is a risk factor for poor prognosis of these diseases (Ndrepepa, 2018; Wannamethee et al., 2018; Yu and Cheng, 2020). Wang et al. (2016) showed that during a follow-up of 2-3 years, when serum UA levels increased in CHD patients who were about 60 years old, the risks of all-cause mortality and cardiac death increased significantly. Baseline serum UA level has been effective in evaluating and predicting the prognosis of patients with different subtypes of ACS (Timóteo et al., 2013; Spiga et al., 2017; Cicero et al., 2018; Lazzeroni et al., 2018; Tscharre et al., 2018). In fact, hyperuricemia is closely related to DM. A high UA level may lead to decreased hepatic insulin clearance rate and insulin sensitivity of target organs, and increased fasting and postprandial insulin levels and hepatic insulin resistance index (Kramer et al., 2010; Perticone et al., 2012; Fiorentino et al., 2018). Serum UA is an independent risk factor and an important predictor of DM (Keerman et al., 2020). However, among the clinical studies on the prognosis of ACS patients with $\mathrm{DM}$, there are few studies on the predictive value of UA, especially with regard to elderly people (who have not been clearly reported), and the pathophysiological mechanism has not been clarified. Our study analyzed the baseline serum UA level after admission and the incidence of main cardiovascular adverse events within 120 months after discharge of elderly ACS patients, aiming to investigate whether serum UA level is an independent risk factor for the longterm prognosis of elderly ACS patients with DM.

\section{Patients and methods}

This was a prospective cohort study conducted in the Chinese People's Liberation Army (PLA) General
Hospital Cardiac Center from January 2006 to December 2012. We enrolled a total of 718 patients aged (81.57士 2.16) years who were hospitalized for coronary angiography due to chest tightness, chest pain, or other suspicious symptoms of ACS, including 509 males and 209 females, 242 diabetic patients and 476 nondiabetic patients. Based on the results of coronary angiograms, the subjects were divided into a control group and a research group; the control group included 89 patients who had normal coronary angiograms, while the research group included 629 patients diagnosed with ACS. The patients were followed up once every 12 months after discharge to record the occurrence of major adverse cardiac event (MACE), including nonfatal acute myocardial infarction (AMI), target vessel revascularization (percutaneous coronary intervention (PCI) or coronary artery bypass grafting (CABG)), cardiac death, and all-cause death. The main outcomes of this study were MACE and all-cause mortality (cardiovascular and non-cardiovascular mortalities).

\subsection{Inclusion criteria}

All patients underwent coronary angiography in Chinese PLA General Hospital to confirm the diagnosis of CHD. The severity of coronary artery stenosis was recorded in terms of Gensini score, and those who recorded experimental data were trained uniformly. Based on coronary angiogram results, individualized treatment strategies were performed for all patients, including intensive treatment with medicine, PCI or CABG; and long-term follow-up was performed after discharge.

\subsection{Exclusion criteria}

The patients who met the following criteria were exclude: patients with severe valvular heart disease, pulmonary hypertension, severe liver insufficiency, rheumatoid arthritis, malignant tumors, gout, or infectious diseases; patients who had taken UA-lowering drugs within the past month; or patients with neuropsychiatric disorders that prevented them from cooperating with the researcher.

\subsection{Clinical data}

We recorded general information (age, gender, body mass index (BMI), heart rate, blood pressure, left ventricular ejection fraction (LVEF), and Gensini score), cardiovascular risk factors (hypertension, hyperlipidemia, 
previous myocardial infarction (MI), previous stroke, chronic renal failure (CRF), and smoking), and blood biochemical indices at admission (total cholesterol (TC), triglyceride (TG), high-density lipoprotein-cholesterol (HDL-C), low-density lipoprotein-cholesterol (LDL-C), fasting blood glucose (FBG), UA, creatinine, and glycosylated hemoglobin $(\mathrm{HbAlc}))$, which were taken after $12 \mathrm{~h}$ of fasting prior to admission. We also recorded cardiovascular medication experience (aspirin, clopidogrel, $\beta$-blockers, angiotensin-converting enzyme inhibitor (ACEI)/angiotensin receptor blocker (ARB), or statins).

The estimated glomerular filtration rate (eGFR) was calculated with the Chinese Modification of Diet in Renal Disease (MDRD) equation (Ma et al., 2006): eGFR $=175 \times$ Scr $^{-1.234} \times$ age $^{-0.179} \times 0.79$ (if female), where eGFR is in $\mathrm{mL} / \mathrm{min}$ per $1.73 \mathrm{~m}^{2}$, Scr is standardized creatinine concentration in $\mathrm{mg} / \mathrm{dL}$, and age is in year. Scr was calculated with a calibration equation (Guo et al., 2014): $\mathrm{Scr}=0.795 \times \operatorname{Scr}_{\mathrm{EM}}+0.29$, where $\mathrm{Scr}_{\mathrm{EM}}$ is Scr measured by enzymatic method (mg/dL). Chronic kidney disease was defined as eGFR $<60 \mathrm{~mL} / \mathrm{min}$ per $1.73 \mathrm{~m}^{2}$.

\subsection{Statistical analysis}

All data in this study were processed using SPSS software Version 25.0 (IBM Corporation, Armonk, NY, USA). The measurement data of normal distribution were expressed as mean \pm standard deviation (SD). If the variances were homogeneous, the $t$-test was used; if the variances were not homogeneous, the rank sum test was used. The measurement data of non-normal distribution were represented by medians with interquartile range (IQR). The enumeration data were expressed numerically and differences between groups were assessed using the Chi-square test. Analysis of variance (ANOVA) was used to compare data between groups. Multiple Cox proportional regression analysis (odds ratio (OR), 95\% confidence interval (CI)) was used to identify the factors associated with all-cause mortality and MACE. $P<0.05$ indicates a statistically significant difference.

\section{Results}

\subsection{Baseline characteristics of study subjects}

Of the 718 subjects in this cohort study, a total of 629 patients diagnosed with ACS were assigned to the study group. After measuring their UA levels according to the third equalization point, we divided them into three groups: Group 1 (low UA levels), including 205 patients with UA levels from 15.7 to $303.0 \mu \mathrm{mol} / \mathrm{L}$; Group 2 (moderate UA levels), including 209 patients with UA levels from 303.4 to $380.8 \mu \mathrm{mol} / \mathrm{L}$; and Group 3 (high UA levels), including 215 patients with UA levels from 381.9 to $912.8 \mu \mathrm{mol} / \mathrm{L}$. The UA level in the control group was normal ((344.2 \pm 98.5$) \mu \mathrm{mol} / \mathrm{L})$.

The average heart rate of Group 3 was higher than that of the control group. LVEF of patients with DM in Group 2 was significantly lower than that of those in Group 1 or the control group $(P<0.01)$. TG of patients with DM in Group 3 was significantly higher than that of those in Group $2(P<0.01)$. In the study group, with the increase of UA level, eGFR showed a significant downward trend, and patients with DM had lower eGFR than patients without $\mathrm{DM}(P<0.01)$, while TG $(P<0.05)$ and FBG $(P<0.01)$ levels of patients with DM were higher than those of patients without $\mathrm{DM}$. There were no statistically significant differences in blood pressure, coronary artery stenosis score, combined diseases, or medication status ( $P>0.05$; Table 1$)$.

Pearson's correlation analysis showed that UA levels were negatively correlated with eGFR $(r=-0.202, P=$ 0.002; Fig. 1a) and EF ( $r=-0.139, P=0.04$; Fig. 1b) in ACS patients with DM.

\subsection{Long-term prognosis of patients}

We conducted long-term follow-up of all 718 enrolled subjects, with a follow-up time of 40-120 months (median: 63 months; IQR: 51-74 months). The allcause mortalities of the four groups with DM were $10.0 \%(2 / 20), 10.6 \%$ (7/66), 39.8\% (33/83), and 58.9\% (43/73) $(P<0.0001)$, respectively; the respective cardiac mortalities were $5.0 \%(1 / 20), 3.0 \%(2 / 66), 18.1 \%(15 /$ $83)$, and $42.5 \%(31 / 73)(P<0.0001)$; MACE rates were $15.0 \%(3 / 20), 21.2 \%(14 / 66), 54.2 \%(45 / 83)$, and $65.8 \%$ $(48 / 73)(P<0.0001)$, respectively. The all-cause mortalities of the four groups without DM were $13.0 \%$ (9/69), 15.1\% (21/139), 19.0\% (24/126), and 47.9\% $(68 / 142)(P<0.0001)$, respectively; the respective cardiac mortalities were $1.4 \%(1 / 69), 4.3 \%$ (6/139), 9.5\% (12/ $126)$, and $32.4 \%(46 / 142)(P<0.0001)$; MACE rates were $13.0 \%$ (9/69), 25.2\% (35/139), 31.0\% (39/126), and $55.6 \%(79 / 142)(P<0.0001)$, respectively. The longterm prognoses of the four groups, both with and without DM, were significantly different $(P<0.0001)$. 
Table 1 Clinical characteristics of octogenarians in study population

\begin{tabular}{|c|c|c|c|c|c|c|c|c|c|}
\hline \multirow[b]{2}{*}{ Characteristics } & \multicolumn{2}{|c|}{ Control group } & \multicolumn{2}{|c|}{ Group 1} & \multicolumn{2}{|c|}{ Group 2} & \multicolumn{2}{|c|}{ Group 3} & \multirow{2}{*}{$\begin{array}{c}P \\
\text { value }\end{array}$} \\
\hline & $\begin{array}{c}\mathrm{DM} \\
(n=20)\end{array}$ & $\begin{array}{c}\text { NDM } \\
(n=69)\end{array}$ & $\begin{array}{c}\mathrm{DM} \\
(n=66)\end{array}$ & $\begin{array}{c}\text { NDM } \\
(n=139)\end{array}$ & $\begin{array}{c}\mathrm{DM} \\
(n=83)\end{array}$ & $\begin{array}{c}\text { NDM } \\
(n=126)\end{array}$ & $\begin{array}{c}\mathrm{DM} \\
(n=73)\end{array}$ & $\begin{array}{c}\text { NDM } \\
(n=142)\end{array}$ & \\
\hline \multicolumn{10}{|l|}{ General conditions } \\
\hline Age (years) & $82.1 \pm 1.8$ & $81.3 \pm 1.7$ & $81.3 \pm 1.7$ & $82.2 \pm 2.4$ & $81.4 \pm 1.9$ & $82.1 \pm 2.3$ & $82.0 \pm 2.2$ & $81.9 \pm 2.0$ & 0.325 \\
\hline Male & 12 & 43 & 37 & 95 & 61 & 100 & 52 & 109 & 0.103 \\
\hline Female & 8 & 26 & 29 & 44 & 22 & 26 & 21 & 33 & 0.211 \\
\hline HR (beat/min) & $66.9 \pm 11.5$ & $71.9 \pm 10.4$ & $75.3 \pm 13.8$ & $74.2 \pm 14.0$ & $74.8 \pm 13.3$ & $72.1 \pm 12.3$ & $77.9 \pm 14.5^{\Delta \Delta}$ & $76.2 \pm 15.5$ & 0.001 \\
\hline BMI $\left(\mathrm{kg} / \mathrm{m}^{2}\right)$ & $24.4 \pm 3.3$ & $24.0 \pm 3.9$ & $24.4 \pm 3.3$ & $24.1 \pm 3.5$ & $25.6 \pm 3.4$ & $24.1 \pm 3.1$ & $24.3 \pm 3.2$ & $24.9 \pm 3.3$ & 0.501 \\
\hline $\mathrm{SBP}(\mathrm{mmHg})$ & $142.5 \pm 21.7$ & $136.7 \pm 17.7$ & $141.8 \pm 13.4$ & $135.4 \pm 22.6^{\S}$ & $138.3 \pm 21.9$ & $136.5 \pm 23.5$ & $138.1 \pm 24.1$ & $135.1 \pm 20.4$ & 0.549 \\
\hline $\mathrm{DBP}(\mathrm{mmHg})$ & $71.6 \pm 14.6$ & $72.9 \pm 11.1$ & $70.5 \pm 11.6$ & $71.1 \pm 11.7$ & $70.7 \pm 12.7$ & $72.6 \pm 11.2$ & $68.4 \pm 12.1$ & $72.4 \pm 11.1^{\S}$ & 0.006 \\
\hline $\mathrm{EF}(\%)$ & $60.1 \pm 7.9$ & $60.7 \pm 6.6$ & $58.1 \pm 6.1$ & $55.6 \pm 13.7$ & $54.1 \pm 8.6^{\Delta \Delta \# \#}$ & $56.6 \pm 8.3$ & $54.7 \pm 11.0$ & $53.6 \pm 10.6$ & 0.001 \\
\hline Gensini score & 0 & 0 & $49.1 \pm 39.6$ & $50.8 \pm 38.2$ & $55.2 \pm 40.8$ & $45.8 \pm 42.3$ & $59.2 \pm 42.4$ & $60.6 \pm 48.7$ & 0.493 \\
\hline \multicolumn{10}{|l|}{ Risk factors } \\
\hline Hypertension & 17 & 55 & 55 & 97 & 75 & 90 & 63 & 109 & 0.641 \\
\hline Hyperlipoidemia & 4 & 19 & 20 & 30 & 18 & 26 & 16 & 33 & 0.566 \\
\hline Previous MI & 0 & 6 & 8 & 27 & 17 & 23 & 10 & 32 & 0.105 \\
\hline Previous stroke & 5 & 7 & 10 & 27 & 21 & 27 & 18 & 32 & 0.441 \\
\hline CRF & 2 & 3 & 7 & 9 & 14 & 12 & 15 & 20 & 0.367 \\
\hline Smoking & 3 & 9 & 17 & 27 & 26 & 31 & 23 & 35 & 0.287 \\
\hline \multicolumn{10}{|l|}{ Baseline blood features } \\
\hline $\mathrm{TC}(\mathrm{mmol} / \mathrm{L})$ & $3.9 \pm 1.0$ & $3.8 \pm 0.8$ & $3.9 \pm 1.0$ & $4.1 \pm 0.9$ & $4.1 \pm 1.0$ & $4.1 \pm 1.0$ & $4.1 \pm 0.89$ & $4.2 \pm 1.0$ & 0.499 \\
\hline $\mathrm{TG}(\mathrm{mmol} / \mathrm{L})$ & $1.3 \pm 0.6$ & $1.2 \pm 0.5$ & $1.5 \pm 0.9$ & $1.2 \pm 0.6^{\S}$ & $1.4 \pm 0.6$ & $1.3 \pm 0.7$ & $1.7 \pm 0.9^{\nabla \nabla}$ & $1.5 \pm 0.8^{\S}$ & 0.003 \\
\hline $\mathrm{HDL}-\mathrm{C}(\mathrm{mmol} / \mathrm{L})$ & $1.3 \pm 0.5$ & $1.2 \pm 0.3$ & $1.2 \pm 0.3$ & $1.2 \pm 0.4$ & $1.1 \pm 0.3$ & $1.2 \pm 0.4$ & $1.1 \pm 0.5$ & $1.1 \pm 0.3$ & 0.297 \\
\hline LDL-C (mmol/L) & $2.1 \pm 0.7$ & $2.1 \pm 0.7$ & $2.1 \pm 0.9$ & $2.5 \pm 0.7^{\S \S}$ & $2.3 \pm 0.9$ & $2.4 \pm 0.8$ & $2.3 \pm 0.7$ & $2.5 \pm 0.9^{\S}$ & 0.003 \\
\hline FBG (mmol/L) & $6.0 \pm 1.7$ & $5.7 \pm 1.6$ & $8.5 \pm 3.1^{\triangle \Delta}$ & $6.3 \pm 2.2^{\S \S}$ & $7.8 \pm 2.6^{\Delta \Delta}$ & $6.3 \pm 2.7^{\S \S}$ & $8.2 \pm 3.0^{\Delta \Delta}$ & $6.5 \pm 2.6^{\S \S}$ & 0.001 \\
\hline HbA1c $(\%)$ & $7.9 \pm 2.6$ & $7.0 \pm 2.8$ & $8.2 \pm 2.7$ & $6.9 \pm 2.3^{\S \S}$ & $8.1 \pm 2.3$ & $7.1 \pm 2.6^{\S \S}$ & $8.4 \pm 2.2$ & $7.1 \pm 2.4^{\S \S}$ & 0.001 \\
\hline $\begin{array}{l}\text { eGFR }(\mathrm{mL} / \mathrm{min} \\
\left.\text { per } 1.73 \mathrm{~m}^{2}\right)\end{array}$ & $68.6 \pm 17.6$ & $78.2 \pm 16.7^{\S}$ & $79.4 \pm 20.8$ & $77.8 \pm 30.1$ & $69.0 \pm 18.5^{\# \#}$ & $71.5 \pm 16.0^{\triangle \Delta \#}$ & $62.2 \pm 20.8^{* * \nabla}$ & $68.5 \pm 19.9^{\S \Delta \Delta^{* * *}}$ & 0.002 \\
\hline \multicolumn{10}{|c|}{ Cardiovascular medications } \\
\hline Aspirin & 19 & 64 & 66 & 137 & 81 & 122 & 71 & 136 & 0.483 \\
\hline Clopidogrel & 15 & 58 & $66^{\Delta \Delta}$ & 138 & $80^{\triangle \Delta}$ & 120 & $67^{\triangle^{*}}$ & 133 & 0.001 \\
\hline$\beta$-Blocker & 14 & 36 & 42 & 83 & 53 & 67 & 56 & 98 & 0.336 \\
\hline ACEI/ARB & 13 & 42 & 41 & 71 & 49 & 59 & 51 & 76 & 0.559 \\
\hline Statin & 15 & 64 & $64^{\Delta \Delta}$ & 129 & 74 & 120 & 66 & 135 & 0.030 \\
\hline
\end{tabular}

Data are shown as mean \pm standard deviation (SD) or number $(n)$ of patients. ${ }^{\triangle} P<0.05,{ }^{\triangle}{ }^{P} P<0.01$, ACS vs. control; ${ }^{\#} P<0.05,{ }^{\#} P<0.01$, Group 2 vs. Group $1 ;{ }^{*} P<0.05,{ }^{* *} P<0.01$, Group 3 vs. Group $1 ;{ }^{\nabla} P<0.05,{ }^{\nabla} P<0.01$, Group 3 vs. Group $2 ;{ }^{\S} P<0.05,{ }^{\S \S} P<0.01$, DM vs. NDM in the same group. $P$ values in bold are $<0.05$. ACEI: angiotensin-converting enzyme inhibitor; ACS: acute coronary syndrome; ARB: angiotensin receptor blocker; BMI: body mass index; CRF: chronic renal failure; DBP: diastolic blood pressure; DM: diabetes mellitus; EF: ejection fraction; eGFR: estimated glomerular filtration rate; FBG: fasting blood glucose; HbAlc: glycosylated hemoglobin; HDL-C: high-density lipoproteincholesterol; HR: heart rate; LDL-C: low-density lipoprotein-cholesterol; MI: myocardial infarction; NDM: non-diabetes mellitus; SBP: systolic blood pressure; TG: triglyceride; TC: total cholesterol.

All-cause mortality and MACE in Group 2 were significantly higher for patients with $\mathrm{DM}(P=0.001)$, but there were no significant differences in the other three groups between DM and non-DM patients for longterm prognosis. All-cause mortality, cardiac mortality, and MACE rates were significantly higher in both DM and non-DM (NDM) patients in Group 3 compared to the other groups $(P<0.01)$. Meanwhile, the all-cause mortality, cardiac mortality, and MACE rates in Group 2 patients with DM were significantly higher than those in the control group or Group $1(P<0.01)$, as shown in Table 2 and Fig. 2.

We analyzed the data with Kaplan-Meier survival curves, and the results showed a statistically significant 
(a)

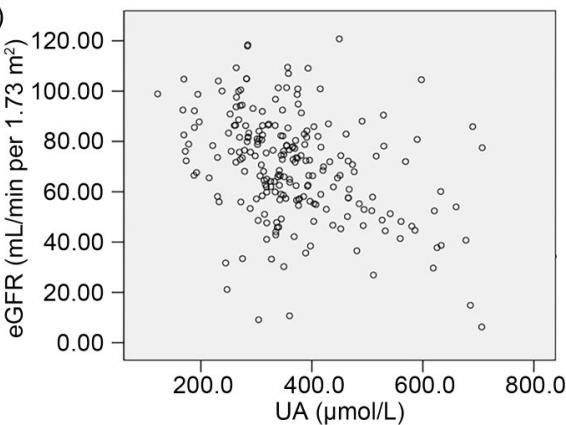

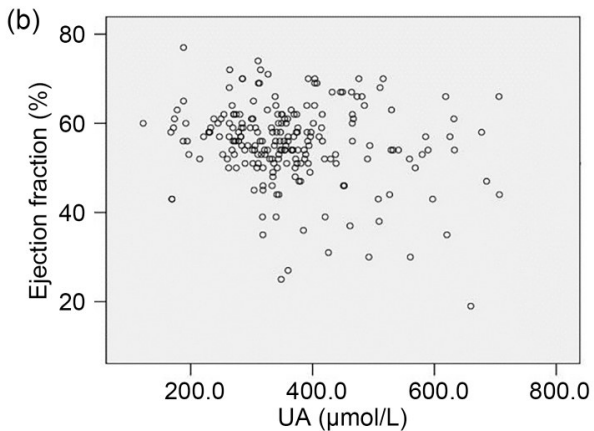

Fig. 1 Correlation of uric acid (UA) with estimated glomerular filtration rate (eGFR) (a) and ejection fraction (b) in acute coronary syndrome (ACS) patients with diabetes mellitus (DM).

Table 2 Long-term prognosis in ACS octogenarians among the four groups

\begin{tabular}{|c|c|c|c|c|c|}
\hline \multirow{2}{*}{ Group } & \multicolumn{5}{|c|}{ Number of patients } \\
\hline & All-cause death & Cardiac death & AMI & Revascularization & MACE \\
\hline \multicolumn{6}{|l|}{ Control } \\
\hline $\mathrm{DM}(n=20)$ & 2 & 1 & 1 & 0 & 3 \\
\hline $\operatorname{NDM}(n=69)$ & 9 & 1 & 0 & 0 & 9 \\
\hline \multicolumn{6}{|l|}{ Group 1} \\
\hline DM $(n=66)$ & 7 & 2 & 2 & 5 & 14 \\
\hline $\operatorname{NDM}(n=139)$ & 21 & 6 & 5 & 9 & 35 \\
\hline \multicolumn{6}{|l|}{ Group 2} \\
\hline $\mathrm{DM}(n=83)$ & $33^{\triangle \# \#}$ & $15^{\# \#}$ & 2 & 10 & $45^{\triangle \triangle \# \#}$ \\
\hline $\operatorname{NDM}(n=126)$ & $24^{\S \S}$ & 12 & 4 & 11 & $39^{\S \S}$ \\
\hline \multicolumn{6}{|l|}{ Group 3} \\
\hline $\mathrm{DM}(n=73)$ & $43^{\triangle \Delta \nabla \nabla * *}$ & $31^{\triangle \Delta \nabla \nabla * *}$ & 0 & 5 & $48^{\triangle \triangle^{* * *}}$ \\
\hline $\operatorname{NDM}(n=142)$ & $68^{\triangle \Delta \nabla \nabla * *}$ & $46^{\triangle \Delta \nabla \nabla * *}$ & 0 & 11 & $79^{\triangle \Delta \nabla \nabla * *}$ \\
\hline$P$-value & $<0.001$ & $<0.001$ & 0.433 & 0.308 & $<0.001$ \\
\hline
\end{tabular}

${ }^{\triangle} P<0.05,{ }^{\triangle}{ }^{\circ} P<0.01$, ACS vs. control group; ${ }^{\sharp} P<0.05,{ }^{\# \#} P<0.01$, Group 2 vs. Group $1 ;{ }^{*} P<0.05,{ }^{* *} P<0.01$, Group 3 vs. Group $1 ;{ }^{\nabla} P<0.05,{ }^{\nabla \nabla} P<0.01$, Group 3 vs. Group $2 ;{ }^{\S} P<0.05,{ }^{\S \S} P<0.01$, DM vs. NDM. ACS: acute coronary syndrome; AMI: acute myocardial infarction; DM: diabetes mellitus; NDM: non-diabetes mellitus; MACE: major cardiovascular events; UA: uric acid.

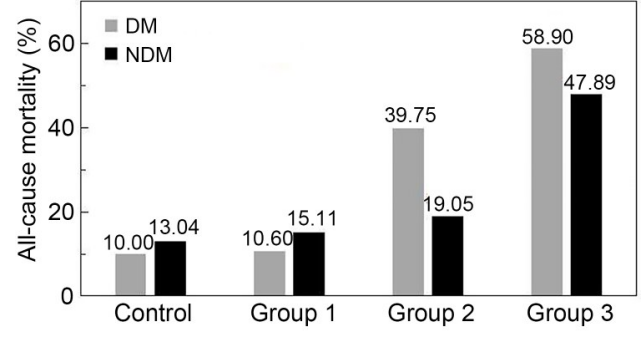

Fig. 2 All-cause mortalities in the four groups for DM and NDM patients. UA levels: control (105.0-488.8 $\mu \mathrm{mol} / \mathrm{L}), \mathrm{DM}$ (366.88 \pm 114.44$) \mu \mathrm{mol} / \mathrm{L}$ and NDM (332.55 \pm 86.24$) \mu \mathrm{mol} / \mathrm{L}$; Group 1 (15.7-303.0 $\mu \mathrm{mol} / \mathrm{L})$, DM (253.47 \pm 44.66$) \mu \mathrm{mol} / \mathrm{L}$ and NDM (252.41 \pm 51.18$) \mu \mathrm{mol} / \mathrm{L}$; Group 2 (303.4-380.8 $\mu \mathrm{mol} / \mathrm{L})$, DM (346.80 \pm 22.22$) \mu \mathrm{mol} / \mathrm{L}$ and NDM (348.57 \pm 23.38$) \mu \mathrm{mol} / \mathrm{L}$; Group $3(381.9-912.8 \mu \mathrm{mol} / \mathrm{L})$, DM $(499.66 \pm 100.73) \mu \mathrm{mol} / \mathrm{L}$ and NDM (486.47 \pm 91.08$) \mu \mathrm{mol} / \mathrm{L}$. DM: diabetes mellitus; NDM: non-diabetes mellitus; UA: uric acid.

difference in long-term survival in ACS patients with $\mathrm{DM}(P=0.001)$. As the UA level increased, the longterm survival rate decreased sharply (Fig. 3).

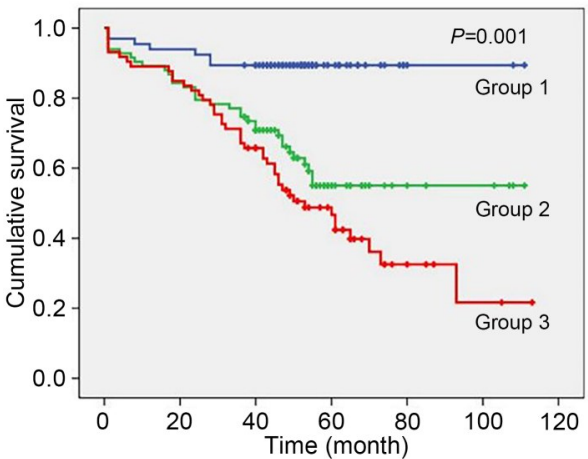

Fig. 3 Kaplan-Meier survival curves of long-term survival rates for the diabetic patient study group.

\subsection{Effects of independent risk factors on long- term prognosis}

At the end of follow-up, we performed a Cox regression analysis to determine the factors that were associated with all-cause mortality and MACE. After 
adjusting for general conditions (sex, age, BMI, systolic blood pressure (SBP), and LVEF), risk factors (hypertension, hyperlipidemia, previous MI, stroke, and $\mathrm{CRF}$ ), and blood biochemistry indicators (TG, TC, HDL-C, and LDL-C), we found that heart rate $(\mathrm{OR}=1.04,95 \% \mathrm{CI}=1.02-1.06, P=0.001)$, eGFR $(\mathrm{OR}=$ $0.983,95 \% \mathrm{CI}=0.965-0.998, P=0.002)$, diastolic blood pressure (OR $=0.968,95 \% \mathrm{CI}=0.945-0.990, P=0.006)$, and UA $(\mathrm{OR}=2.106,95 \% \mathrm{CI}=1.244-3.568, P=0.006)$ were independent risk factors for all-cause mortality in ACS patients with DM. In addition, heart rate $(\mathrm{OR}=$ $1.03,95 \% \mathrm{CI}=1.01-1.05, P=0.001)$ and $\mathrm{UA}(\mathrm{OR}=$ $1.752,95 \% \mathrm{CI}=1.068-2.876, P=0.026)$ were independent risk factors for MACE in ACS patients with DM.

\subsection{Diagnostic powers of uric acid for long-term all-cause death}

We established receiver operating characteristic (ROC) curves corresponding to the survival and death conditions of the DM group and the NDM group (Figs. 4a and 4b), and calculated the cutoff value of UA. The area under curve (AUC) of UA in the DM group was $0.726(95 \% \mathrm{CI}=0.658-0.794, P=0.0001$; Fig. 4a) and the cutoff value for all-cause mortality was $353.6 \mu \mathrm{mol} / \mathrm{L}$ (sensitivity: $67.4 \%$; specificity: $65.7 \%$ ). The AUC of UA in the NDM group was $0.663(95 \% \mathrm{CI}=$ $0.601-0.726, P=0.0001 ;$ Fig. $4 \mathrm{~b})$. Then, according to these UA cutoff values, we divided DM patients in the study group into two groups, Group A (UA $\leqslant 353.6 \mu \mathrm{mol} / \mathrm{L})$ and Group B $(\mathrm{UA}>353.6 \mu \mathrm{mol} / \mathrm{L})$. The all-cause mortality rates were $23.97 \%(29 / 121)$ in Group A and $53.47 \%(54 / 101)$ in Group B $(P<0.001)$. Thus, the

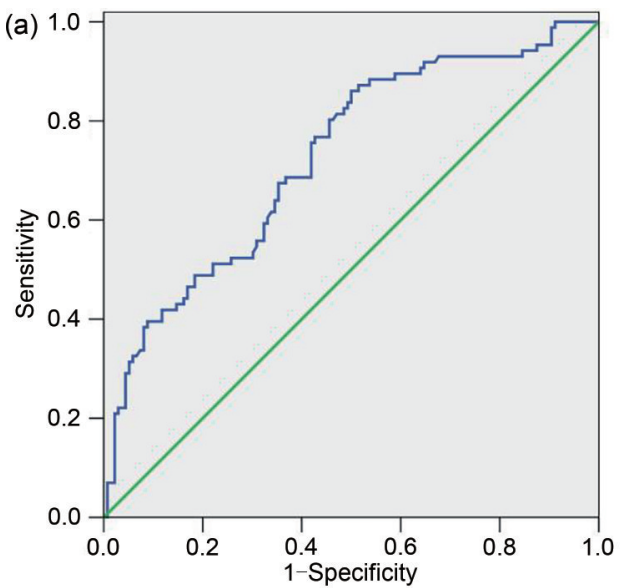

statistical results showed that elevated baseline of serum UA level was an independent risk factor for all-cause mortality in elderly ACS patients with DM.

\section{Discussion}

ACS is a generic term for all kinds of clinical syndromes caused by acute or subacute myocardial ischemia. The main pathophysiological basis is thrombus formation secondary to coronary artery spasm or rupture of atherosclerotic plaque in coronary arteries (Tsujita et al., 2016; Mani et al., 2019). Traditional risk factors of CHD include age, hypertension, DM, hyperlipidemia, and smoking, and as a major risk factor, DM has been of interest to many scholars (Pullinger et al., 2021). Studies have shown that long-term hyperglycemia leads to increased vascular endothelial permeability and abnormal expression of inflammatory factors, and thus causes the body to be in a state of high inflammatory response (Ruszkowska-Ciastek et al., 2015; Zhou et al., 2020). In addition, hyperglycemia leads to excessive production of reactive oxygen species (ROS) and decreases the activity of antioxidant enzymes, and thus causes oxidative stress (Contreras-Zentella et al., 2019). All of these factors contribute to the development of coronary atherosclerosis, which ultimately leads to the occurrence of CHD. Studies illustrate that as an independent risk factor of $\mathrm{CHD}$, DM has a high predictive value for the occurrence of CHD (Lu et al., 2020). The most common type of DM is type 2. In this study, we selected elderly ACS patients with type $2 \mathrm{DM}$ as research

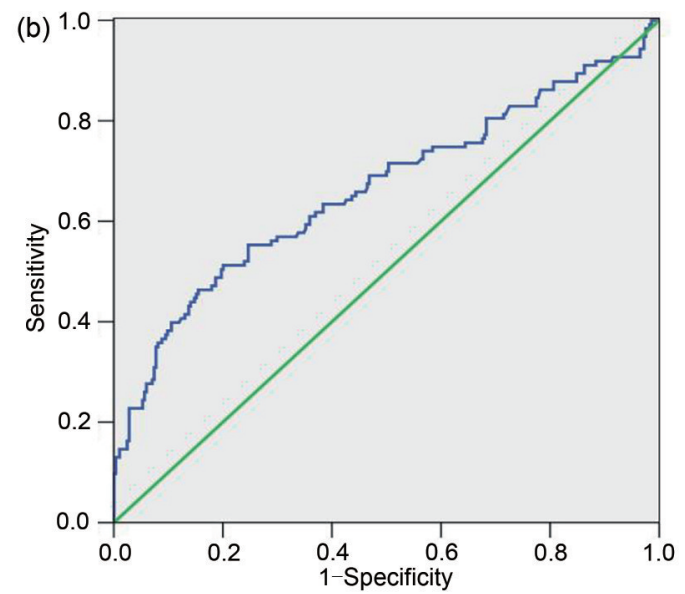

Fig. 4 ROC curves of UA for discrimination between surviving and deceased patients, for ACS patients with DM (a) and NDM (b). ROC: receiver operating characteristic; UA: uric acid; ACS: acute coronary syndrome; DM: diabetes mellitus; NDM: non-diabetes mellitus. 
subjects, which means that the results could be widely applicable. We analyzed the general conditions and prognostic indicators of both DM and NDM patients. Of the elderly patients with ACS included in our study, more than one-third had DM, a $20 \%$ higher rate than previously reported (Sethi et al., 2012).

UA is the final metabolite of various purines in the body, and the UA level of the body is affected by a variety of factors such as excessive purine intake, reduced excretion, metabolic disorders, and gene mutations, which may increase the UA level (Bailey, 2019; Zhao et al., 2021). Gertler et al. (1951) first proposed an association between baseline serum UA level and CHD. Since then, multiple epidemiological studies and metaanalyses have shown that hyperuricemia is an independent risk factor for CHD. Baseline serum UA level is positively correlated with the incidence and severity of CHD, and for each $1 \mathrm{mg} / \mathrm{dL}$ increase in UA level, patient's chances of all-cause mortality increase by approximately $12 \%$ (Purnima and El-Aal, 2016). However, there is no clear report on the indicators for longterm prognosis in elderly ACS patients with DM, using $\mathrm{UA}$ as an evaluation factor.

In this study, we prospectively collected the clinical information of 718 elderly patients with suspected ACS who were admitted due to chest tightness and chest pain, and performed ten years of follow-up. Our objective was to analyze the relationship between baseline serum UA levels and long-term prognosis, including allcause mortality, cardiovascular death, and MACE, and our emphasis was on the long-term prognosis of patients with DM. The main findings of our study are as follows: (1) UA is a predictor of all-cause mortality and MACE in elderly ACS patients with DM; (2) the ability of UA to predict the prognosis of these patients increases with higher UA levels; (3) with the extension of followup time, the effect of UA on the prognosis of these patients becomes more significant; (4) the predictive power of UA is stronger in DM patients than in NDM patients. In addition, we found that in the study group, the Gensini scores of patients did not show significant differences as UA levels increased, which was inconsistent with previous reports (Levantesi et al., 2013; Wei et al., 2017). This finding may be related to the significant increase in the coronary artery lesion scores in all elderly patients enrolled in this study. By comparison and analysis of all-cause mortality and MACE rate in the study group, we found that only Group 2 showed differences in the results of DM and NDM patients, while high UA made no difference in Group 3. However, the long-term prognosis indicators of both $\mathrm{DM}$ and NDM patients in Group 3 were significantly worse than those of the other three groups. These results suggest that the impact of elevated UA level on the long-term prognosis of elderly ACS patients may be higher than that of diabetes.

Our results also showed that the UA cutoff value for elderly ACS patients with DM was $353.6 \mu \mathrm{mol} / \mathrm{L}$. Cox regression results showed that after adjusting for other factors, UA was indeed an independent risk factor for all-cause mortality $(\mathrm{OR}=2.106, P=0.006)$ and MACE $(\mathrm{OR}=1.752, P=0.026)$ in elderly ACS patients with $\mathrm{DM}$. Therefore, in clinical practice, we should pay attention not only to the traditional risk factors for CHD such as age, hypertension, hyperlipidemia, smoking, or drinking, but also to patients' UA levels, and physicians should consider the effect of drugs on the UA metabolism.

Previous research indicated that a hyperuricemic state in the body mainly promotes the development of CHD via the following means: (1) activating platelets, promoting thrombosis, and activating inflammatory mediators to reduce plaque stability (Kimura et al., 2020); (2) stimulating nicotinamide adenine dinucleotide phosphate (NADPH) oxidase, leading to abnormal structure and function of vascular endotheliocytes, and affecting production of adenosine triphosphate (ATP), resulting in dysfunction of vascular endotheliocytes (Grossman et al., 2019); (3) leading to the precipitation of urate crystals and excessive generation of oxygen free radicals, which reduces the stability of plaques (Wang et al., 2019). Saito et al. (2019) pointed out that elevated UA level was closely related to vascular endothelial dysfunction in ACS patients, which may contribute to poor prognosis. In addition, UA reduces the ability of endotheliocytes to utilize nitric oxide (NO), and activates the oxidative stress response of mitochondria and the renin-angiotensin-aldosterone system (RAAS), thus increasing the incidence of cardiovascular disease (Nishida et al., 2013). In terms of the interaction between UA and DM, inflammation and oxidative stress induced by high UA may further reduce insulin sensitivity and affect the expression of insulin genes in patients (Verma et al., 2020). UA increases intracellular ectonucleotide pyrophosphatase/phosphodiesterase 1 (ENPP1), thereby directly inhibiting the insulin signaling pathway at the receptor level (Tassone et al., 2018). In 
ACS patients with DM, UA causes insulin dysfunction and abnormal glucose metabolism, promoting oxidative stress, and ultimately aggravates the damage caused to the cardiovascular system by hyperglycemia itself.

In summary, with the growth of global aging and the increasing incidence of CHD, the population with CHD complicated with DM is increasing year by year. In addition to developing diagnostic techniques and treatment methods, it is necessary to find appropriate and accessible predictors to evaluate the prognosis of these patients. At present, there are relatively few studies on predictors of prognosis for elderly ACS patients with DM. This study used large samples, performed an up-toten-year follow-up study, and finally confirmed that UA is an independent risk factor for evaluating the prognosis of such patients. Moreover, the UA test process is simple and inexpensive. Therefore, it can be used as an addition to the clinical detection index.

This study has the following limitations: (1) sample size: although a total of 718 elderly ACS patients were included in this study, there were only $20 \mathrm{DM}$ patients in the control group and $73 \mathrm{DM}$ patients in the high-UA group, so additional verification may be required in these two groups; (2) it was a single-center observational study, and selectivity bias may have affected the results, which should be verified by multi-center studies; (3) the purpose of the study was to research the correlation between UA and the prognosis of elderly ACS patients with DM, but the experimental data did not include mean data of blood glucose, LDL-C, or UA from the follow-up period.

\section{Conclusions}

Baseline serum UA level is an independent predictor of long-term all-cause death, cardiac death, and MACE in elderly ACS patients with DM, and the higher the UA level, the more accurate the prediction. Therefore, UA may become a new indicator for the long-term prognosis of elderly ACS patients with DM.

\section{Acknowledgments}

We thank the patient advisers for the information they provided.

\section{Author contributions}

Zhenhong FU and Qing XI planned the study, and wrote and edited the manuscript. Yang JIAO and Jihang WANG performed the experimental research and data analysis, and wrote and edited the manuscript. Mingzhi SHEN, Hao XUE, Wei DONG, and Jun GUO conducted a survey, and provided samples and other logistics support. Qing XI, Xia YANG, and Yundai CHEN contributed to the drafting and participated in research discussion. All authors have read and approved the final manuscript, and therefore, have full access to all the data in the study and take responsibility for the integrity and security of the data.

\section{Compliance with ethics guidelines}

Yang JIAO, Jihang WANG, Xia YANG, Mingzhi SHEN, Hao XUE, Jun GUO, Wei DONG, Yundai CHEN, Qing XI, and Zhenhong FU declare that they have no conflict of interest.

The study was performed in accordance with the ethical standards laid down in the 1964 Declaration of Helsinki and its later amendments and was approved by the Ethics Service Center of the Chinese PLA General Hospital in China. Written informed consent was obtained from all participants.

\section{References}

Bailey CJ, 2019. Uric acid and the cardio-renal effects of SGLT2 inhibitors. Diabetes Obes Metab, 21(6):1291-1298. https://doi.org/10.1111/dom.13670

Bonaca MP, Gutierrez JA, Cannon C, et al., 2018. Polyvascular disease, type 2 diabetes, and long-term vascular risk: a secondary analysis of the IMPROVE-IT TRIAL. Lancet Diabetes Endocrinol, 6(12):934-943. https://doi.org/10.1016/S2213-8587(18)30290-0

Cicero AFG, Fogacci F, Giovannini M, et al., 2018. Serum uric acid predicts incident metabolic syndrome in the elderly in an analysis of the Brisighella Heart Study. Sci Rep, 8:11529. https://doi.org/10.1038/s41598-018-29955-w

Contreras-Zentella ML, Sánchez-Sevilla L, Suárez-Cuenca JA, et al., 2019. The role of oxidant stress and gender in the erythrocyte arginine metabolism and ammonia management in patients with type 2 diabetes. PLOS ONE, 14(7): e0219481. https://doi.org/10.1371/journal.pone.0219481

Fiorentino TV, Sesti F, Succurro E, et al., 2018. Higher serum levels of uric acid are associated with a reduced insulin clearance in non-diabetic individuals. Acta Diabetol, 55(8):835-842. https://doi.org/10.1007/s00592-018-1153-8

Fu ZH, Xue H, Guo J, et al., 2013. Long-term prognostic impact of cystatin $\mathrm{C}$ on acute coronary syndrome octogenarians with diabetes mellitus. Cardiovasc Diabetol, 12:157. https://doi.org/10.1186/1475-2840-12-157

Gertler MM, Garn SM, Levine SA, 1951. Serum uric acid in relation to age and physique in health and in coronary heart disease. Ann Intern Med, 34(6):1421-1431. https://doi.org/10.7326/0003-4819-34-6-1421

Grossman C, Grossman E, Goldbourt U, 2019. Uric acid variability at midlife as an independent predictor of coronary heart disease and all-cause mortality. PLoS ONE, 14(8): $\mathrm{e} 0220532$.

https://doi.org/10.1371/journal.pone.0220532

Guo XX, Wang Y, Wang K, et al., 2018. Stability of a type 2 
diabetes rat model induced by high-fat diet feeding with low-dose streptozotocin injection. J Zhejiang Univ-Sci B (Biomed \& Biotechnol), 19(7):559-569. https://doi.org/10.1631/jzus.B1700254

Guo XZ, Qin Y, Zheng K, et al., 2014. Improved glomerular filtration rate estimation using new equations combined with standardized cystatin $\mathrm{C}$ and creatinine in Chinese adult chronic kidney disease patients. Clin Biochem, 47(13-14): 1220-1226. https://doi.org/10.1016/j.clinbiochem.2014.05.060

Jin DY, Liu CL, Tang JN, et al., 2017. Interleukin-18, matrix metalloproteinase-22 and -29 are independent risk factors of human coronary heart disease. $J$ Zhejiang Univ-Sci B (Biomed \& Biotechnol), 18(8):685-695. https://doi.org/10.1631/jzus.B1700073

Keerman M, Yang F, Hu H, et al., 2020. Mendelian randomization study of serum uric acid levels and diabetes risk: evidence from the Dongfeng-Tongji cohort. BMJ Open Diabetes Res Care, 8:e00834. https://doi.org/10.1136/bmjdrc-2019-000834

Kimura Y, Yanagida T, Onda A, et al., 2020. Soluble uric acid promotes atherosclerosis via AMPK (AMP-activated protein kinase)-mediated inflammation. Arterioscler Thromb Vasc Biol, 40(3):570-582. https://doi.org/10.1161/ATVBAHA.119.313224

Komaru Y, Takeuchi T, Suzuki L, et al., 2019. Recurrent cardiovascular events in patients with newly diagnosed acute coronary syndrome: influence of diabetes and its management with medication. $J$ Diabetes Complications, 34(3):107511. https://doi.org/10.1016/j.jdiacomp.2019.107511

Kramer CK, von Mühlen D, Jassal SK, et al., 2010. A prospective study of uric acid by glucose tolerance status and survival: the Rancho Bernardo Study. J Intern Med, 267(6):561-566. https://doi.org/10.1111/j.1365-2796.2009.02168.x

Lazzeroni D, Bini M, Camaiora U, et al., 2018. Serum uric acid level predicts adverse outcomes after myocardial revascularization or cardiac valve surgery. Eur J Prev Cardiol, 25(2):119-126. https://doi.org/10.1177/2047487317744045

Levantesi G, Marfisi RM, Franzosi MG, et al., 2013. Uric acid: a cardiovascular risk factor in patients with recent myocardial infarction. Int J Cardiol, 167(1):262-269. https://doi.org/10.1016/j.ijcard.2011.12.110

Lu TY, Forgetta V, Yu OHY, et al., 2020. Polygenic risk for coronary heart disease acts through atherosclerosis in type 2 diabetes. Cardiovasc Diabetol, 19:12. https://doi.org/10.1186/s12933-020-0988-9

Ma YC, Zuo L, Chen JH, et al., 2006. Modified glomerular filtration rate estimating equation for Chinese patients with chronic kidney disease. J Am Soc Nephrol, 17(10): 2937-2944. https://doi.org/10.1681/ASN.2006040368

Mani P, Puri R, Schwartz GG, et al., 2019. Association of initial and serial C-reactive protein levels with adverse cardiovascular events and death after acute coronary syndrome: a secondary analysis of the VISTA-16 trial. JAMA Cardiol,
4(4):314-320.

https://doi.org/10.1001/jamacardio.2019.0179

National Center for Cardiovascular Diseases, 2021. Annual report on cardiovascular health and diseases in China (2020). $J$ Cardiovasc Med (Hagerstown), 3:276 (in Chinese).

Ndrepepa G, 2018. Uric acid and cardiovascular disease. Clinica Chimica Acta, 484:150-163. https://doi: 10.1016/j.cca.2018.05.046

Nishida Y, Takahashi Y, Susa N, et al., 2013. Comparative effect of angiotensin II type I receptor blockers on serum uric acid in hypertensive patients with type 2 diabetes mellitus: a retrospective observational study. Cardiovasc Diabetol, 12:159. https://doi.org/10.1186/1475-2840-12-159

Perticone F, Sciacqua A, Perticone M, et al., 2012. Serum uric acid and 1-h postload glucose in essential hypertension. Diabetes Care, 35(1):153-157. https://doi.org/10.2337/dc11-1727

Pullinger CR, O'Connor PM, Naya-Vigne JM, et al., 2021. Levels of prebeta-1 high-density lipoprotein are a strong independent positive risk factor for coronary heart disease and myocardial infarction: a meta-analysis. $J$ Am Heart Assoc, 10(7):e018381. https://doi.org/10.1161/JAHA.120.018381

Purnima S, El-Aal BGA, 2016. Serum uric acid as prognostic marker of coronary heart disease (CHD). Clin Investig Arterioscler, 28(5):216-224 https://doi.org/10.1016/j.arteri.2016.05.006

Ruszkowska-Ciastek B, Sokup A, Wernik T, et al., 2015. Effect of uncontrolled hyperglycemia on levels of adhesion molecules in patients with diabetes mellitus type 2. J Zhejiang Univ-Sci B (Biomed \& Biotechnol), 16(5):355-361. https://doi.org/10.1631/jzus.B1400218

Saito Y, Kitahara H, Nakayama T, et al., 2019. Relation of elevated serum uric acid level to endothelial dysfunction in patients with acute coronary syndrome. $J$ Atheroscler Thromb, 26(4):362-367. https://doi.org/10.5551/jat.45179

Sethi SS, Akl EG, Farkouh ME, 2012. Diabetes mellitus and acute coronary syndrome: lessons from randomized clinical trials. Curr Diab Rep, 12(3):294-304. https://doi.org/10.1007/s11892-012-0272-9

Spiga R, Marini MA, Mancuso E, et al., 2017. Uric acid is associated with inflammatory biomarkers and induces inflammation via activating the NF- $\mathrm{KB}$ signaling pathway in HepG2 cells. Arterioscler Thromb Vasc Biol, 37(6):12411249. https://doi.org/10.1161/ATVBAHA.117.309128

Tassone EJ, Cimellaro A, Perticone M, et al., 2018. Uric acid impairs insulin signaling by promoting ENPP1 binding to insulin receptor in human umbilical vein endothelial cells. Front Endocrinol (Lausanne), 9:98. https://doi.org/10.3389/fendo.2018.00098

Timóteo AT, Lousinha A, Labandeiro J, et al., 2013. Serum uric acid: a forgotten prognostic marker in acute coronary syndromes? Eur Heart J Acute Cardiovasc Care, 2(1): 44-52. https://doi.org/10.1177/2048872612474921 
Tscharre M, Herman R, Rohla M, et al., 2018. Uric acid is associated with long-term adverse cardiovascular outcomes in patients with acute coronary syndrome undergoing percutaneous coronary intervention. Atherosclerosis, 270: 173-179. https://doi.org/10.1016/j.atherosclerosis.2018.02.003

Tsujita K, Yamanaga K, Komura N, et al., 2016. Lipid profile associated with coronary plaque regression in patients with acute coronary syndrome: subanalysis of PRECISEIVUS trial. Atherosclerosis, 251:367-372. https://doi.org/10.1016/j.atherosclerosis.2016.05.025

Verma S, Ji QH, Bhatt DL, et al., 2020. Association between uric acid levels and cardio-renal outcomes and death in patients with type 2 diabetes: a subanalysis of EMPA-REG OUTCOME. Diabetes Obes Metab, 22(7):1207-1214. https://doi.org/10.1111/dom.13991

Wang CW, Yan WL, Wang H, et al., 2019. APOE polymorphism is associated with blood lipid and serum uric acid metabolism in hypertension or coronary heart disease in a Chinese population. Pharmacogenomics, 20(14):1021-1031. https://doi.org/10.2217/pgs-2019-0048

Wang RJ, Song YX, Yan YQ, et al., 2016. Elevated serum uric acid and risk of cardiovascular or all-cause mortality in people with suspected or definite coronary artery disease: a meta-analysis. Atherosclerosis, 254:193-199. https://doi.org/10.1016/j.atherosclerosis.2016.10.006

Wannamethee SG, Papacosta O, Lennon L, et al., 2018. Serum uric acid as a potential marker for heart failure risk in men on antihypertensive treatment: the British Regional Heart Study. Int J Cardiol, 252:187-192. https://doi.org/10.1016/j.ijcard.2017.11.083

Wei XB, Jiang L, Liu YH, et al., 2017. Serum uric acid as a simple risk factor in patients with rheumatic heart disease undergoing valve replacement surgery. Clin Chim Acta, 472: 69-74.

https://doi.org/10.1016/j.cca.2017.07.019

Xu RJ, Kong WM, An XF, et al., 2020. Physiologically-based pharmacokinetic-pharmacodynamics model characterizing CYP2C19 polymorphisms to predict clopidogrel pharmacokinetics and its anti-platelet aggregation effect following oral administration to coronary artery disease patients with or without diabetes. Front Pharmacol, 11:593982. https://doi.org/10.3389/fphar.2020.593982

Yu W, Cheng JD, 2020. Uric acid and cardiovascular disease: an update from molecular mechanism to clinical perspective. Front Pharmacol, 11:582680. https://doi.org/10.3389/fphar.2020.582680

Zhao ZL, Zhao YS, Zhang YQ, et al., 2021. Gout-induced endothelial impairment: the role of SREBP2 transactivation of YAP. FASEB J, 35(6):e21613. https://doi.org/10.1096/fj.202100337R

Zhou X, Li JQ, Wei LJ, et al., 2020. Silencing of DsbA-L gene impairs the PPAR $\gamma$ agonist function of improving insulin resistance in a high-glucose cell model. J Zhejiang Univ-Sci B (Biomed \& Biotechnol), 21(12):990-998. https://doi.org/10.1631/jzus.B2000432 